\title{
Données relatives aux blessures en Colombie-Britannique : opinion des décideurs en ce qui concerne le transfert de connaissances
}

C Mitton, Ph.D. (1, 2); YC MacNab, Ph.D. (2,3); N Smith, M.A. (1); L Foster, Ph.D. (4)

\section{Résumé}

Les décideurs provinciaux et régionaux du domaine de la prévention des blessures de la Colombie-Britannique ont été interrogés sur ce qu'ils croient être la meilleure manière de transférer ou de diffuser des données pertinentes. Ces décideurs ( $\mathrm{n}=13$ ) ont mentionné que les données devraient appuyer le processus décisionnel en dressant un tableau complet de la situation. Ils croient que les renseignements sur les types et les taux de blessures devraient non seulement être liés aux déterminants et aux causes, mais aussi aux répercussions et aux conséquences. Selon eux, une telle chaîne de données est nécessaire pour planifier et évaluer les mesures de promotion de la santé. Les décideurs croient également que les fournisseurs de données doivent investir plus d'efforts pour améliorer leur capacité de collecte de données dans le but de permettre aux professionnels du domaine des blessures de mieux comprendre, d'interpréter et d'utiliser les données. Les commentaires recueillis, qui peuvent facilement être utilisés par d'autres administrations ou secteurs de politique, permettent de mieux saisir la valeur concrète du transfert et de l'échange de connaissances entre les chercheurs et les décideurs.

\section{Mots-clés : fardeau des blessures, transfert de connaissances et utilisation des connaissances}

\section{Introduction}

Malgré tous les efforts qui ont été et qui seront déployés pour recueillir des données sur les répercussions et le fardeau des maladies chroniques au Canada, la manière optimale d'utiliser ces données pour élaborer des politiques et améliorer la pratique demeure incertaine. Le concept de transfert de connaissances ${ }^{i}$ consiste à transmettre des renseignements aux décideurs en favorisant la participation interactive. Au cours des dix dernières années, les chercheurs et les décideurs ont déterminé les facteurs qui favorisent le transfert des connaissances ou qui y nuisent ${ }^{1-5}$. À la suite de leurs travaux, des directives concernant les mécanismes et les stratégies de mise en œuvre efficace du transfert de connaissances ont été publiées $^{6-7}$. Les recommandations les plus courantes consistaient à établir une collaboration continue entre les chercheurs et les décideurs ${ }^{6,8,9}$, à adopter une attitude appropriée, à promouvoir les valeurs et la culture, à favoriser le renforcement des capacités au sein des organismes de soins de santé ${ }^{3}$ et à assurer une communication claire et sans délai en utilisant un langage compris par le public cible 2,6,10 $^{2}$ Il demeure toutefois important de fournir davantage de données probantes en ce qui concerne les pratiques de transfert de connaissances afin de veiller à ce que les efforts de recherche et de collecte de données soient gérés adéquatement. Les études de cas empiriques, dont l'ensemble de données provient de contextes particuliers (tels que celui qui nous concerne), devraient favoriser la compréhension et l'amélioration immédiate des pratiques. Les résultats de la présente étude, qui intéresseront particulièrement les personnes travaillant dans les domaines de la prévention des blessures et de la promotion de la santé, peuvent également servir au transfert de connaissances portant sur des maladies chroniques et d'autres contextes de soins de santé.

\section{Méthodologie}

La collecte de données a été effectuée à l'aide d'entrevues semi-structurées menées auprès de 13 décideurs ayant des connaissances directes sur la prévention des blessures en Colombie-Britannique. Le protocole d'entrevue est joint en annexe. Les membres du groupe étudié provenaient des organismes suivants : le ministère de la Santé de la Colombie-Britannique (récemment divisé en deux ministères, le ministère des Services de santé et le ministère

i Pour obtenir de plus amples renseignements, veuillez consulter le site : http://www.researchpolicy.ca/whatwehavelearned/develop_approach.asp (en anglais seulement). Les termes connexes comprennent les suivants : application des connaissances, échange de connaissances, utilisation des connaissances, diffusion de la recherche. 
de la Vie saine et du Sport), la Provincial Health Services Authority (PHSA) et deux des cinq régies régionales de la santé. Les entrevues ont été effectuées auprès de cadres supérieurs et intermédiaires occupant notamment des postes de directeur général, de directeur, de gestionnaire ou de responsable de projet. Ces cadres sont habituellement responsables de la planification, de l'établissement des priorités ou de l'évaluation des fonctions. Les entrevues ont été menées en juin et en juillet 2005.

Les responsables de l'entrevue ont fourni des échantillons de données à chacun des participants aux fins d'observation (p. ex. tableau 1, tableau 2 et schéma 1). Ils ont ensuite demandé aux participants de leur préciser les sources de données auxquelles ils ont habituellement accès et de leur fournir des commentaires à l'égard des échantillons de données. Chacune des entrevues a été enregistrée (avec la permission du participant) et transcrite. L'analyse a été effectuée à l'aide de la méthode de comparaison continue et les thèmes et les sous-thèmes ont été déterminés de manière inductive. Les commentaires rédigés par les responsables de l'entrevue ont constitué une source de renseignements supplémentaire. Le Behavioural Research Ethics Board de l'Université de la Colombie-Britannique a fourni une approbation déontologique.

\section{Environnement politique concernant la prévention des blessures en Colombie-Britannique}

Les cinq régies régionales de la santé sont chargées d'élaborer et de mettre en œuvre des plans et des programmes de prévention des blessures. Elles doivent déterminer dans quelle mesure accorder la priorité à la prévention des blessures plutôt qu'à d'autres initiatives, évaluer la pertinence et l'efficacité des options de programme, inviter les autres secteurs à participer (au besoin) et évaluer le succès, les coûts relatifs et les avantages liés à leurs efforts. Les régies régionales de la santé n'en sont pas toutes au même stade en ce qui concerne l'élaboration de la politique de prévention des blessures. La PHSA, qui appuie les activités de cueillette et d'analyse de données et les activités d'application des connaissances est une source importante de données sur la sécurité des patients, notamment en ce qui concerne les événements iatrogènes médicamenteux, les infections nosocomiales et les blessures causées par la radiation.

Le gouvernement de la ColombieBritannique, qui doit en premier lieu assurer la gérance, effectue également des activités de surveillance et de contrôle. La Health Autority Division du ministère des Services de santé discute actuellement d'ententes de rendement avec les régies régionales de la santé. Ces ententes comprennent des points de repère relatifs aux chutes généralement élaborés dans le but d'inciter les régies à rendre des comptes sur les résultats atteints à l'aide des ressources qui leur ont été allouées. La Healthy Children, Women and Seniors Branch (récemment déménagée pour appuyer la création du nouveau ministère de la Vie saine et du Sport), de pair avec les recommandations de l'agent de santé provincial, constitue le principal centre d'élaboration de politiques et de conseils $\mathrm{du}$ gouvernement provincial en ce qui a trait aux blessures.

L'agent de santé provincial, qui travaille désormais pour le ministère des Services de santé et le ministère de la Vie saine et du Sport, a aidé les régies régionales de la santé à élaborer les points de repère relatifs aux chutes et a préparé un rapport spécial sur les chutes chez les aînés qui 1) souligne l'ampleur de la question, 2) évalue les répercussions sur le système de soins de santé et 3) fournit des recommandations de stratégies de prévention fondées sur des données probantes ${ }^{12}$. Évidemment, chacune des régies régionales de la santé tient compte de sa propre situation. Des activités de recherche et des collectes additionnelles, qui appuient l'élaboration de recommandations stratégiques, sont réalisées depuis 1997 par la Injury Research and Prevention Unit (BCIRPU) qui est située dans le Children's and Women's Health Centre of British Columbia. Ces activités sont effectuées en sous-traitance depuis le démantèlement de l'Office of Injury Prevention du Ministère en 2001. La BCIRPU accroît ses sources de données en établissant des ententes avec la Commission des accidents du travail (connue sous le nom de WorkSafeBC), Statistique Canada, l'Institut canadien d'information sur la santé (ICIS), l'Insurance Corporation of British Columbia (ICBC), la Gendarmerie royale du Canada (GRC) et le service ambulancier de la Colombie-Britannique.

D’autres principaux intervenants (p. ex. la Croix-Rouge et la GRC) participent au moyen de différents mécanismes, tels que l'Injury Prevention Leadership Network de la Colombie-Britannique, un comité directeur provincial qui a pour objectif de fournir des directives et des conseils en ce qui concerne les orientations stratégiques générales. Toutefois, d'autres organisations existent également, notamment l'Aboriginal Health Network, le Sport and Injury-Free Advisory Committee et la Falls Prevention Coalition de la Colombie-Britannique. La Healthy Living Alliance de la Colombie-Britannique, un consortium d'organisations dont le travail porte sur les principales maladies chroniques, appuie également les questions liées au mode de vie sain, y compris la prévention des blessures. La province, la PHSA et les régies régionales de la santé sont conscientes des intérêts de ces trois groupes, mais oublient parfois leur rôle et les liens qu'ils entretiennent avec les autres intervenants. En bref, l'environnement politique relatif à la prévention des blessures en Colombie-Britannique est plutôt fragmenté; il est composé de ministères, d'agences et d'organisations multiples.

\section{Données sur les blessures utilisées dans les recherches}

Composantes de cet environnement politique, des chercheurs de l'Université de la Colombie-Britannique mènent actuellement une étude de cas dans le but d'élaborer un ensemble de méthodes statistiques qui permettra de recueillir des données plus précises sur les blessures survenues dans la province. Cette étude est réalisée dans le cadre d'un programme de recherche continue intitulé Burden of Injury in $B C$ and Its Local Communities: Information and Evidence for Communitybased Prevention Strategy, Health Policy and Service Provision (dont le titre abrégé est Burden of Injury in BC), qui est financé par les Instituts de recherche en santé 
du Canada. Le programme vise principalement 1) à élaborer une synthèse des méthodes analytiques dans le but d'établir un cadre de recherche systématique sur le fardeau lié aux blessures qui comprendra des activités de surveillance et de contrôle spatio-temporel, d'évaluation du fardeau et des risques, de diffusion des recherches et d'application des connaissances; et 2) à favoriser l'utilisation de ces méthodes au cours de l'examen du fardeau lié à la mortalité et aux invalidités causées par des blessures en Colombie-Britannique et au sein de ses collectivités ${ }^{13-14}$.

$\mathrm{Au}$ cours des entrevues réalisées auprès des décideurs, des spécimens de tableaux portant sur le profil du fardeau des blessures de chacune des régies régionales de la santé et de l'ensemble de la Colombie-Britannique ont été présentés aux participants. Les spécimens de tableaux comprenaient des données sur les comptes et les taux de mortalité et d'hospitalisation des cinq principales causes de blessures, classées selon le sexe. Ils ont été établis à partir des données administratives sur les populations recueillies de 1991 à 2000 13 -14 . Les tableaux 1 et 2 présentent des statistiques sur le fardeau des blessures en fonction des causes, qui ont été mesurées en nombre d'années de vie perdues en raison d'un décès prématuré (AVPDP), en années de vie vécues avec une invalidité (AVVI) et en années de vie corrigées du facteur invalidité (AVCI) ${ }^{15}$. Ces mesures du fardeau des blessures ont été établies à l'aide des données sur la mortalité et les hospitalisations causées par des blessures recueillies, de 1991 à 2000, auprès des cinq régies régionales de la santé et dans l'ensemble de la Colombie-Britannique ${ }^{14}$. Les estimations d'AVPDP, d'AVVI et d'AVCI des tableaux 1 et 2 ont été établies à partir des estimations du poids et de la durée des invalidités présentées dans l'étude sur la charge mondiale de la morbidité réalisée en 1990, dans le cadre de laquelle aucune pondération en fonction de l'âge n'a été effectuée et un taux d'actualisation annuel de $3 \%$ a été établi ${ }^{14-15}$. Il est à noter que l'AVCI est une mesure de "l'écart de santé » qui permet de combiner les répercussions liées à la mortalité et à la morbidité et de les évaluer simultanément. Mises au point dans le cadre de l'étude sur la charge mondiale de la morbidité15, les mesures d'AVCI ont été élaborées à titre d'indicateurs de santé de la population dans les évaluations de la santé publique et de "monnaie de mesure » dans les analyses coût-efficacité liées à l'établissement des priorités et à l'évaluation des interventions en matière de santé ${ }^{14-15}$.

Deux spécimens de cartes, qui fournissaient des données sur les risques annuels de blessures iatrogènes chez les enfants et les adolescents âgés de 1 à 19 ans dans les 16 zones desservies par les services de santé et constituant les subdivisions géographiques des régies de la santé, ont été présentés aux participants. L'une des cartes, incluse dans le schéma 1, met en relief l'AVCI des risques élevés et faibles des blessures iatrogènes chez les personnes de sexe masculin. Des estimations bayésiennes des risques annuels relatifs, quantifiés par les quotients des taux d'AVCI sur la moyenne de la Colombie-Britannique, ont été réalisées pour préparer cette carte $\mathrm{e}^{13,16}$. Une zone desservie par les services de santé était perçue comme ayant un risque de blessures iatrogènes élevé (ou faible) si l'estimation d'intervalle de $95 \%$ (c'est-àdire les limites supérieures et inférieures) du risque relatif était supérieure (ou inférieure) à un ${ }^{13,16}$.

\section{Résultats}

Les résultats des entrevues contiennent des renseignements sur le contenu et les processus qui devraient permettre aux chercheurs et aux décideurs de la Colombie-Britannique de diffuser efficacement les données relatives aux blessures et d'y accéder. Le contenu de l'ensemble de données doit dresser un tableau complet des causes et des conséquences. Les processus de diffusion devraient cibler des populations précises et tenir compte des capacités variées des personnes et des organisations d'utiliser les renseignements fournis.

\section{Contenu de l'information}

Bien que les participants connaissaient les mesures d'AVCI et les avaient déjà utilisées pour effectuer de la planification en santé, l'un d'entre eux a mentionné : « [nous] ne les utilisons probablement pas autant que nous le devrions, mais nous les utilisons tout de même ». [No3] [Traduction] Toutefois, les participants croient que d'autres groupes ne connaissent pas aussi bien cette mesure et qu'ils seraient plus à l'aise avec des indicateurs tels que les taux de morbidité et d'hospitalisation. L'un des participants a mentionné que les mesures d'AVCI peuvent ne pas être aussi intéressantes que les autres mesures puisqu'elles ne sont pas liées ou ne correspondent pas aux « objectifs stratégiques du système de santé ». [ $\left.\mathrm{N}^{\circ} 1\right]$ [Traduction] À la différence de leur responsabilité envers les objectifs stratégiques, les gestionnaires du système de santé de la Colombie-Britannique ne sont pas responsables des changements apportés à cette mesure en vertu de l'accord de rendement conclu avec la province. Il semble adéquat d'affirmer que les obstacles institutionnels ou systémiques influencent grandement le processus de transfert des connaissances. Les résultats présentés ci-dessous corroborent cette affirmation.

Les décideurs souhaitent avoir accès à des données détaillées de manière à saisir l'entière complexité des questions stratégiques. Ils veulent obtenir une " chaîne " de données qui leur permettrait d'examiner les circonstances précédant et succédant les blessures. Beaucoup de données concrètes et descriptives sont disponibles en ce qui concerne l'incidence et la prévalence des blessures - les types de blessure, les données démographiques concernant les personnes blessées. Ces données sont utiles, mais les décideurs souhaitent également obtenir des renseignements sur les déterminants et les circonstances des blessures, c'est-à-dire des renseignements qui leur permettront de comprendre la raison d'être des situations survenant au sein de leur collectivité et de vérifier si des préoccupations, des situations ou des besoins sont propres à leur collectivité et si des mesures doivent être adoptées.

Les décideurs souhaitent également étudier les données sous différents angles et examiner les conclusions relatives aux blessures à la lumière d'un éventail de variables. Les données démographiques des personnes touchées, le moment et le lieu de l'accident et les conditions de l'environnement social ou physique comptent parmi les facteurs qui pourraient favoriser la compréhension et l'explication 
des cas de blessures survenant dans les collectivités. Les enquêtes rétrospectives permettraient sûrement de recueillir ce type de renseignement et d'obtenir une description de chacun des incidents.

" Ce qui m’intéresserait serait d'obtenir des renseignements sur les types de chutes, les causes, les répercussions et les personnes les plus à risque. Donc, nous devons tout d'abord savoir qui sont les personnes susceptibles de tomber, à quel moment elles risquent de tomber et ce qu'elles font lorsqu'elles tombent. » [ $\left.\mathrm{N}^{\circ} 1\right]$ [Traduction]

« Nous savons que les blessures causées par des accidents automobiles sont les plus fréquentes, mais nous devons en savoir davantage. Qu'est-ce que cela veut dire? S'agit-il de conducteurs du secteur commercial, d'état d'ébriété au volant ou de la non-utilisation de la ceinture de sécurité? » [№ 3] [Traduction]

De plus, les données relatives aux déterminants et au contexte permettront aux planificateurs de déterminer les moyens les plus efficaces d'intervenir pour résoudre le problème et organiser des activités d'évaluation. Les participants, qui sont particulièrement intéressés par ces moyens, souhaitent obtenir des données qui les aideront à planifier efficacement et à organiser des activités d'évaluation.

« Je crois qu'il s'agit de préciser et de comprendre les questions et les déterminants et d'établir l'efficacité des solutions. Parce que les solutions seront sans aucun doute fondées sur la compréhension des déterminants. » [ $\mathrm{N}^{\circ}$ 1] [Traduction]

"Quand je pense aux données, je ne pense pas aux taux. J'établis toujours le lien entre les interventions fondées sur des données probantes et les données connexes ainsi que les membres de ma collectivité et les données à leur sujet. Je détermine alors l'intervention la plus efficace possible. C'est tellement important d'établir le lien entre les données sur la santé et les interventions efficaces fondées sur des données probantes. » [No 9] [Traduction]
Les décideurs souhaitent également obtenir des données sur les répercussions des blessures. La cueillette de telles données nécessiterait qu'un suivi des cas de blessure soit effectué et que des renseignements sur les conséquences à moyen et à long termes soient recueillis au sujet des particuliers et de la population en général (y compris des renseignements relatifs au coût total). Les résultats de "l'analyse de rentabilisation » de l'enquête sur la prévention des blessures dépendront de la bonne connaissance des répercussions.

" Connaître les répercussions consiste à savoir si les personnes blessées se rétabliront, si elles retourneront au travail, si elles seront capables de marcher à nouveau ou si leur blessure provoquera, comme on l'observe souvent chez les aînés, la détérioration de la santé. Vous savez, nous pouvons parler du programme ActNow [une initiative de promotion de la santé du gouvernement provincial], nous pouvons parler de modes de vie sains et d'exercices et ainsi de suite, mais il demeure qu'un tel revers est la plupart du temps extrêmement néfaste pour la santé des aînés. » [ $\mathrm{N}^{\circ} 1$ ] [Traduction]

" En ce qui concerne les décisions stratégiques, il est vraiment nécessaire de se servir des données pour effectuer une analyse de rentabilisation et préparer un modèle d'analyse de rentabilisation [pour les investissements relatifs aux interventions de prévention]. Nous essayons de convaincre le gouvernement qu'il faut s'attarder à la question, parce que le fardeau des maladies sur le système n'est vraiment qu'un substitut aux dépenses actuelles du système de santé. Je propose donc d'utiliser ces renseignements et de les convertir en quelque chose que les décideurs qui répartissent les ressources pourront comprendre et interpréter, quelque chose qui leur montrera que les investissements réalisés à une extrémité du continuum permettront de réduire la pression exercée à l'autre extrémité. Voilà un argument convaincant. Si ces décideurs connaissaient le pourcentage des dépenses liées aux blessures réellement engendré par des blessures sportives ou des blessures causées par des noyades ou des brulures, ils pourraient davantage cibler leurs interventions. » [ $\mathrm{N}^{\circ} 2$ ] [Traduction]

Toutefois, selon les participants, les renseignements qui composeront la chaîne de données sont difficiles, voire même impossibles, à obtenir en Colombie-Britannique.

\section{Processus de diffusion des données}

Plusieurs commentaires touchent directement le processus de diffusion. Un éventail d'idées sur la manière la plus efficace de transmettre aux décideurs les renseignements contenus dans les données sur les indicateurs a été fourni par les participants (p. ex. l'utilisation d'un diffuseur de connaissances). Tous les participants ont insisté sur l'importance de la crédibilité des diffuseurs de connaissances et la nécessité d'adapter les connaissances transmises aux publics cibles. Par exemple, ceux qui établissent les priorités peuvent avoir des besoins relatifs aux coûts et aux répercussions qui diffèrent des besoins des personnes responsables de l'évaluation détaillée des programmes.

" Cela dépend vraiment de la raison pour laquelle elles sont utilisées et à qui elles seront envoyées. D’un point de vue décisionnel, si vous comprenez ce qu'elles signifient, alors c'est tout ce qui compte. Il suffit alors d'examiner des données ou des tableaux contenant des données brutes et de recueillir les renseignements requis pour prendre la décision. C'est seulement lorsqu'une justification raisonnée, une analyse de rentabilisation ou quelque chose dans le genre doit être préparé qu'il est nécessaire d'avoir accès à l'ensemble des outils. » [No 5] [Traduction]

La représentation visuelle des données sur une carte semblait plaire aux personnes ayant moins d'expérience (p. ex. décideurs faisant partie du conseil d'administration ou des cadres supérieurs). «Si vous souhaitez communiquer ces renseignements à grande échelle, aux politiciens, aux praticiens ou à toutes les personnes qui doivent les comprendre, je crois que les cartes constituent le moyen le plus efficace. » [ $\left.\mathrm{N}^{\circ} 1\right]$ [Traduction] Les cartes sont perçues comme 
le moyen le plus rapide de transmettre des renseignements à un public qui dispose de peu de temps et qui n'est pas en mesure de lire de longs rapports ou des articles de revues spécialisées ou qui est réticent. La représentation graphique des données est comparable aux notes d'information et aux fiches de renseignements transmises par la Fondation canadienne de la recherche sur les services de santé (www.chsrf.ca) dans le but de joindre les principaux décideurs grâce à des recherches fondées sur des données probantes.

Les cartes sont perçues comme des moyens particulièrement efficaces de présenter des comparaisons. "Vous pouvez regarder les renseignements fournis sur la carte et vous comparer aux autres. C'est bien de pouvoir utiliser des images pour vérifier comment on s'en sort comparativement au reste de la province. " [ $\left.\mathrm{N}^{\circ} 7\right]$ [Traduction] Il est intéressant de mentionner que l'un des participants du gouvernement provincial a abordé la question de comparaison en discutant de l'intention de son ministère d'encourager la compétition entre les autorités en matière de santé.
« J'aime l'idée de représenter graphiquement les données sur les blessures. Je pense que c'est la bonne manière de faire les choses. Cela permet de stimuler la compétition entre les autorités du domaine de la santé. Il faudrait donc utiliser les cartes pour montrer quelles sont les autorités qui ont le taux de blessures et le risque relatif pour certaines blessures les plus élevés de toute la province. " [ $\left[\begin{array}{ll}\mathrm{N}^{\circ} & 3\end{array}\right]$ [Traduction]

Les participants s'entendent pour dire que les cartes sont d'excellents outils de communication et qu'elles servent à faire valoir efficacement un point de vue. "Si je dois utiliser des données pour communiquer quelque chose à quelqu'un, je vais me servir d'une représentation graphique. " [ $\mathrm{N}^{\circ}$ 6] [Traduction] Bien sûr, l'analyse et l'interprétation sont inhérentes aux questions touchant les données.
« Les représentations graphiques sont vraiment efficaces pour communiquer son point de vue. Les données sont présentées en contexte et sont accompagnées d'arguments. Elles font partie d'un ensemble qui tente de démontrer quelque chose. Les représentations graphiques peuvent être très utiles lorsqu'il s'agit d'expliquer un point de vue parce qu'elles fournissent un contexte. » [No 9] [Traduction]

Finalement, les participants ont mentionné que l'efficacité des données sur les blessures, peu importe la manière de les présenter, dépend des capacités des organisations (p. ex. régies régionales de la santé). Parmi celles-ci, on compte les connaissances personnelles et les compétences des gestionnaires de données, des analystes des politiques et des responsables chargés de l'élaboration de programmes et les systèmes nécessaires permettant à ces personnes d'appliquer leurs connaissances et d'influer sur la pratique.

TABLEAU 1

Années de vie perdues attribuables à un décès prématuré (AVPDP), années de vie vécues avec une invalidité (AVVI) et années de vie corrigées du facteur invalidité (AVCI) chez les citoyens de sexe masculin de la Colombie Britannique, classées par autorité en matière de santé et par cause de blessure, total cumulé pour la période de 1991 à 2000.

\begin{tabular}{|c|c|c|c|c|c|c|}
\hline \multirow{3}{*}{ Blessure } & \multicolumn{6}{|c|}{ Personnes de sexe masculin } \\
\hline & \multicolumn{5}{|c|}{ Régies régionales de la santé } & \multirow[b]{2}{*}{ C.-B. } \\
\hline & $\begin{array}{l}\text { Intérieur } \\
\text { de la C.-B. }\end{array}$ & Fraser & $\begin{array}{c}\text { Côte de } \\
\text { Vancouver }\end{array}$ & $\begin{array}{c}\text { Île de } \\
\text { Vancouver }\end{array}$ & $\begin{array}{l}\text { Nord de } \\
\text { la C.-B. }\end{array}$ & \\
\hline \multicolumn{7}{|c|}{ Années de vie vécues avec une invalidité } \\
\hline Blessures causées par un accident de la route & 8500 & 10096 & 5489 & 5951 & 4656 & 34692 \\
\hline Blessures causées par l'utilisation d'autres moyens de transport & 1691 & 1424 & 607 & 1039 & 1186 & 5946 \\
\hline Chutes & 3848 & 5526 & 3630 & 3564 & 2037 & 18604 \\
\hline Brûlures causées par le feu ou de l'eau bouillante & 1121 & 1370 & 1119 & 982 & 1033 & 5624 \\
\hline Noyades & 174 & 336 & 181 & 141 & 120 & 952 \\
\hline Blessures sportives & 562 & 766 & 467 & 470 & 275 & 2540 \\
\hline Autres blessures accidentelles & 8622 & 9826 & 5921 & 7118 & 6088 & 37576 \\
\hline Suicide ou blessures auto-infligées & 1008 & 1925 & 1183 & 1104 & 602 & 5822 \\
\hline Autres blessures intentionnelles & 1219 & 2126 & 1687 & 1290 & 1116 & 7438 \\
\hline
\end{tabular}


TABLEAU 1 (suite)

Années de vie perdues attribuables à un décès prématuré (AVPDP), années de vie vécues avec une invalidité (AVVI) et années de vie corrigées du facteur invalidité (AVCI) chez les citoyens de sexe masculin de la Colombie Britannique, classées par autorité en matière de santé et par cause de blessure, total cumulé pour la période de 1991 à 2000.

\begin{tabular}{|c|c|c|c|c|c|c|}
\hline \multirow{3}{*}{ Blessure } & \multicolumn{6}{|c|}{ Personnes de sexe masculin } \\
\hline & \multicolumn{5}{|c|}{ Régies régionales de la santé } & \multirow[b]{2}{*}{ C.-B. } \\
\hline & $\begin{array}{l}\text { Intérieur } \\
\text { de la C.-B. }\end{array}$ & Fraser & $\begin{array}{c}\text { Côte de } \\
\text { Vancouver }\end{array}$ & $\begin{array}{c}\text { île de } \\
\text { Vancouver }\end{array}$ & $\begin{array}{l}\text { Nord de } \\
\text { la C.-B. }\end{array}$ & \\
\hline \multicolumn{7}{|c|}{ Années de vie perdues attribuables à un décès prématuré } \\
\hline Blessures causées par un accident de la route & 19008 & 19357 & 9565 & 9962 & 11081 & 68973 \\
\hline Blessures causées par l'utilisation d'autres moyens de transport & 2781 & 2794 & 1904 & 2350 & 2914 & 12743 \\
\hline Chutes & 3295 & 4284 & 5125 & 3702 & 1042 & 17448 \\
\hline Brûlures causées par le feu ou de l'eau bouillante & 1112 & 968 & 912 & 720 & 830 & 4542 \\
\hline Noyades & 2024 & 2130 & 1781 & 1905 & 1337 & 9177 \\
\hline Blessures sportives & 138 & 78 & 233 & 28 & 64 & 541 \\
\hline Autres blessures accidentelles & 2009 & 1550 & 1189 & 1595 & 1255 & 7598 \\
\hline Suicide ou blessures auto-infligées & 16335 & 21106 & 19110 & 15252 & 7861 & 79664 \\
\hline Autres blessures intentionnelles & 2420 & 4764 & 4720 & 2116 & 2021 & 16040 \\
\hline \multicolumn{7}{|c|}{ Années de vie corrigées du facteur invalidité } \\
\hline Blessures causées par un accident de la route & 27508 & 29452 & 15054 & 15913 & 15737 & 103664 \\
\hline Blessures causées par l'utilisation d'autres moyens de transport & 4473 & 4218 & 2510 & 3389 & 4099 & 18689 \\
\hline Empoisonnement & 5994 & 18269 & 27127 & 10249 & 3289 & 64928 \\
\hline Chutes & 7143 & 9810 & 8755 & 7266 & 3079 & 36053 \\
\hline Brûlures causées par le feu ou de l'eau bouillante & 2233 & 2338 & 2031 & 1701 & 1863 & 10166 \\
\hline Suicide ou blessures auto-infligées & 17343 & 23031 & 20293 & 16356 & 8463 & 85486 \\
\hline Autres blessures intentionnelles & 3638 & 6890 & 6407 & 3406 & 3137 & 23478 \\
\hline
\end{tabular}


TABLEAU 2

Années de vie perdues attribuables à un décès prématuré (AVPDP), années de vie vécues avec une invalidité (AVVI) et années de vie corrigées du facteur invalidité (AVCI) chez les citoyens de sexe féminin de la Colombie Britannique, classées par autorité en matière de santé et par cause de blessure, total cumulé pour la période de 1991 à 2000.

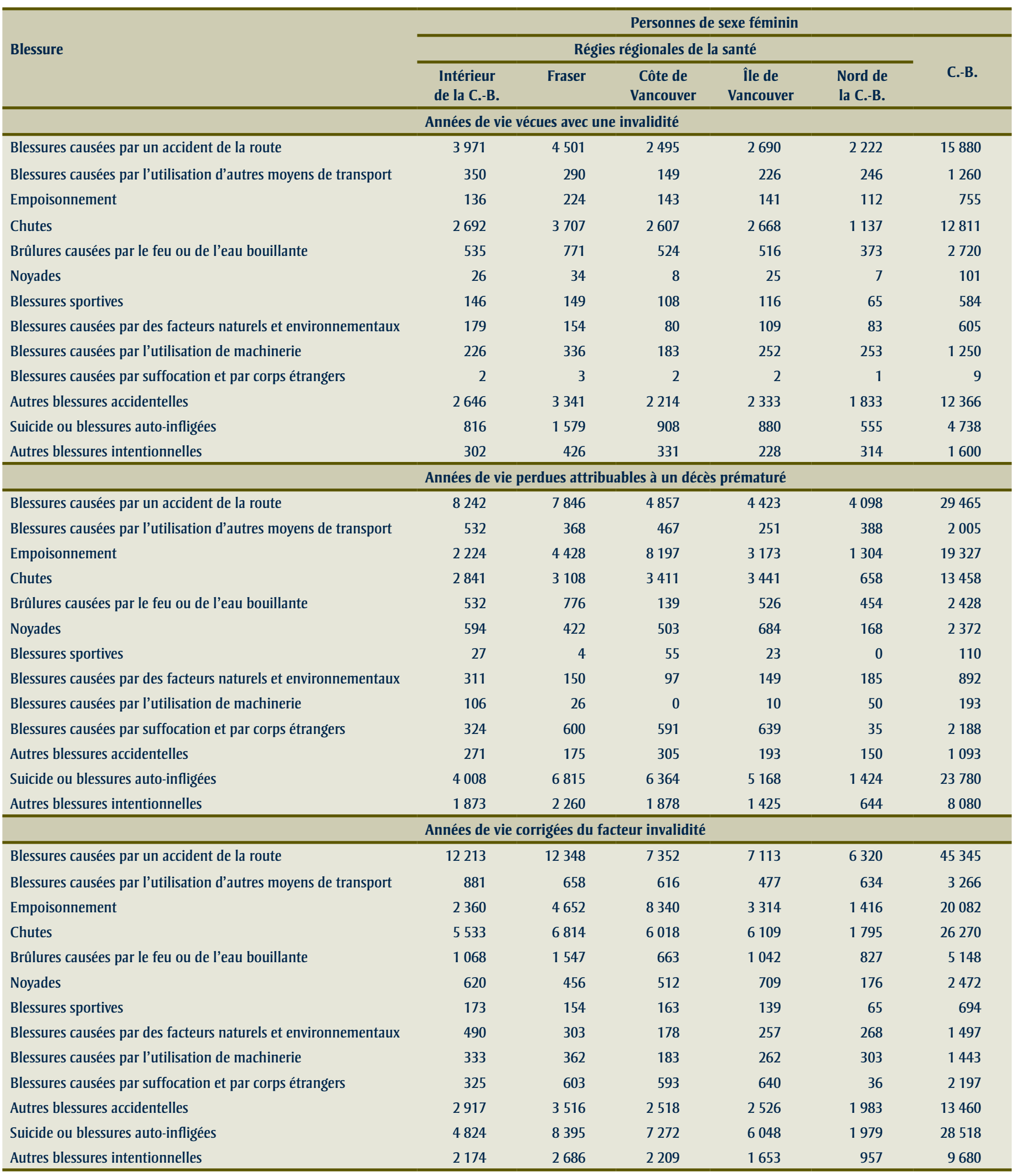




\section{SCHÉMA 1}

Risques relatifs, élevés et faibles, de blessures iatrogènes observées chez les enfants et les adolescents de sexe masculin de la
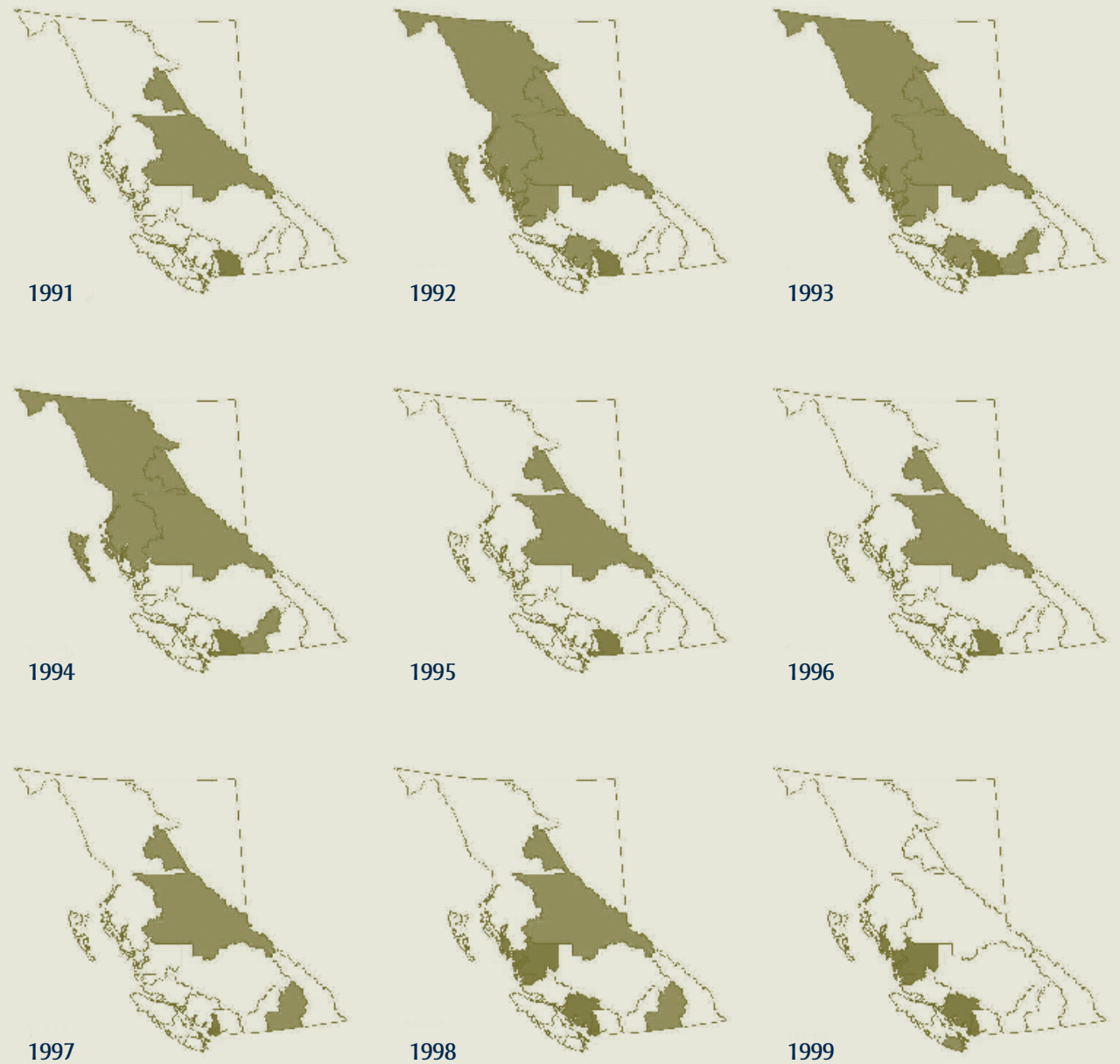

1997

1998

1999

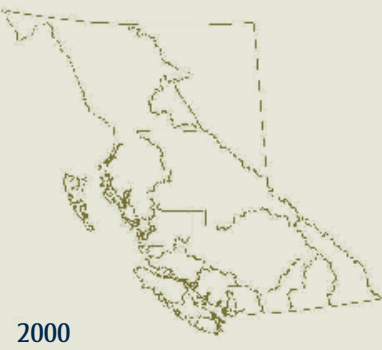

Risque relatif élevé

Risque relatif faible

\section{Risques relatifs}




\section{SCHÉMA 2}

«Chaîne de données » relatives aux blessures

\section{Renseignements} sur les déterminants Renseignements sur les blessures et les circonstances des blessures
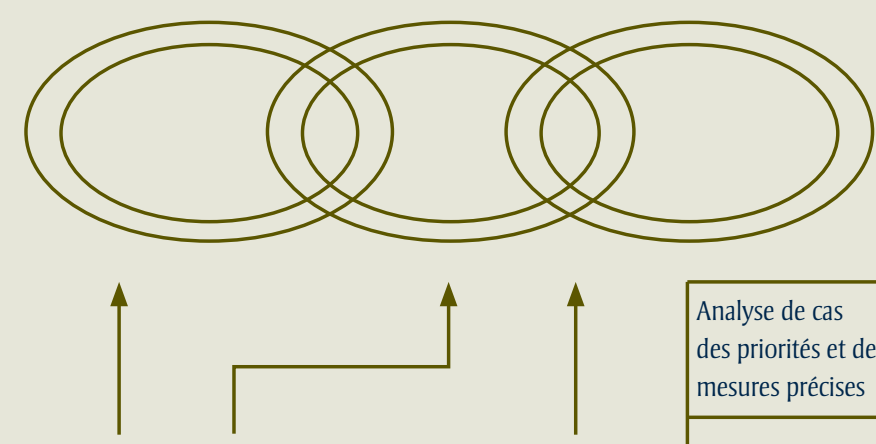

Renseignements servant à la planification et à la mise en ouvre des interventions

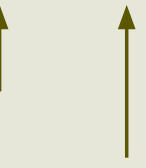

Renseignements provenant de l'évaluation des interventions
Renseignements sur les répercussions et les conséquences des blessures Analyse de cas des priorités et des mesures précises

\section{Discussion}

Il est nécessaire que la recherche et les données correspondent aux besoins des décideurs si l'on veut assurer un transfert de connaissances efficace. Les chercheurs qui décrivent les événements passés ou présents mettent l'accent sur ce qui s'est produit. Les décideurs souhaitent toutefois obtenir des explications et des prévisions : ils veulent connaître les causes et les conséquences possibles ${ }^{17}$. Voilà probablement pourquoi les décideurs du domaine de la prévention des blessures de la Colombie-Britannique insistent sur l'importance de connaître les déterminants des blessures et les résultats probables et escomptés des interventions des régies régionales de la santé.

L'approche qui suit est proposée à la suite de la présente enquête. Pour qu'il y ait un transfert des connaissances dans un certain contexte, les chercheurs doivent être prêts à recueillir des données et à les présenter dans divers formats, des formats qui tiendraient compte des besoins et des capacités des divers décideurs. Dans ce cas, les participants ont approuvé l'utilité d'une représentation visuelle pour les membres de la haute direction. Les données doivent être organisées selon les diverses étapes qui constituent le processus d'élaboration des politiques et le cycle de planification, notamment l'établissement du calendrier, la formulation des politiques, le processus décisionnel et l'application et l'évaluation des politiques ${ }^{18-19}$. Lorsqu'ils organisent les ensembles de données, les chercheurs doivent tenir compte des tâches essentielles au travail des décideurs du système de santé (établissement des priorités et évaluation), de manière à s'adapter à leurs besoins et à les appuyer. Il ne faut toutefois pas oublier que les chercheurs, qui ont eux-mêmes des contraintes de temps et d'argent, n'ont pas toujours accès aux données dont ils ont besoin.

Accéder à ces renseignements permettra aux intervenants d'établir des liens entre les déterminants, la situation, les interventions et les résultats. Fournir une vision plus complexe et complète de la situation va à l'encontre des habitudes des chercheurs et des experts qui ont plutôt tendance à présenter de nombreux renseignements détaillés au sujet d’un événement bien précis. S’il peut être plus coûteux de recueillir de telles données et plus complexes de les analyser, il est possible que des compromis soient faits.
Tel qu'il a été mentionné dans divers ouvrages $^{4,9,20}$, la participation des décideurs aux toutes premières étapes de l'élaboration de la recherche et de la détermination des besoins en matière de données est très importante. Les décideurs seront plus aptes à comprendre et même à utiliser les rapports de données publiées, qui leur paraîtront plus pertinents, s'ils ont participé aux premières étapes de leur élaboration ${ }^{21-22}$.

Finalement, les participants ont clairement indiqué que les renseignements comme tels ne suffisent pas à influencer le contexte politique : les renseignements doivent s'intégrer dans une structure qui peut les contenir et les produire. À ce jour, beaucoup de recherches ont mis l'accent sur l'échange personnalisé de connaissances entre les chercheurs et les utilisateurs ${ }^{23}$. Bien que cet échange est important, les résultats de la présente recherche, qui corroborent ceux de diverses études récentes sur le transfert de connaissances, indiquent que les structures et les systèmes organisationnels peuvent déterminer à quel point les données probantes et les données seront utiles pour répondre aux objectifs des politiques sur la promotion de la santé23-25.

Le schéma 2 résume les résultats de la présente étude à l'aide d'un diagramme. Les données sur les blessures qui intéressent les décideurs de la Colombie-Britannique, constituent une chaîne de renseignements. L'interaction entre les différents éléments de la chaîne a été mise en relief. Cette manière de présenter le transfert de connaissances favorise une nouvelle compréhension du domaine. Le processus décisionnel n'est toutefois pas aussi linéaire que le schéma l'indique. Les déterminants des blessures peuvent influencer les répercussions et les conséquences - les aînés qui risquent davantage de se blesser dans certaines situations, notamment dans les établissements de soins prolongés, peuvent présenter des résultats différents et moins satisfaisants en raison de leur âge et de leur fragilité. Des décisions appropriées devront tenir compte de telles interactions et s'en inspirer. Il sera nécessaire de mener une analyse à plusieurs niveaux, bien qu'une représentation améliorée du contexte réel où surviennent les blessures (indispensable à l'élaboration d'interventions stratégiques 
adéquates) nécessitera une capacité de traitement de l'information accrue de la part des décideurs. L'un des objectifs continus du programme de recherche Burden of Injury in $B C$ consiste à explorer et à élaborer des méthodes de transfert de connaissances qui favorisent la communication efficace des résultats analytiques complexes et l'utilisation de renseignements pertinents pour les questions stratégiques et prioritaires liées à la surveillance, à la prévention et aux interventions.

La petite taille de l'échantillon constitue l'une des principales limites méthodologiques de la présente recherche. Un plus grand nombre de participants aurait possiblement permis de recueillir davantage de points de vue et de mieux comprendre les questions fondamentales liées aux données sur le transfert de connaissances et le fardeau des blessures. Cela dit, la stratégie d'échantillonnage utilisée consistait à rassembler des décideurs de diverses organisations assumant différentes fonctions et responsabilités touchant l'élaboration de politiques et de programmes sur la prévention des blessures. Les points de vue recueillis étaient sensiblement les mêmes. Toutefois, aucun gestionnaire de données (employé des régies régionales de la santé susceptible de manipuler et d'interpréter les ensembles de données précis) ne faisait partie du groupe à l'étude. D’autres études portant sur l'exercice de la politique sur l'intervention des blessures mises en œuvre par les régies régionales de la santé pourraient servir à comprendre de quelle manière les renseignements sont utilisés et pourraient déterminer si les ensembles de données détaillées requis par les participants de la présente étude seront utiles ou si les décideurs réagissent simplement à l'incertitude et à l'anxiété en demandant davantage de renseignements et en repoussant le moment de prendre des décisions difficiles.

D’autres recherches seront nécessaires pour comprendre et expliquer pourquoi la chaîne de données requise pour le processus décisionnel peut ne pas être pertinente à l'environnement politique actuel de la Colombie-Britannique. Est-ce la fragmentation qui pose des difficultés, ou bien les systèmes de collecte de données?
Serait-il utile de créer des dossiers de santé électroniques accessibles dans l'ensemble du système de santé? Préparer une réforme systémique basée sur des besoins antérieurement déterminés (tels que ceux cernés dans le cadre de la présente étude) serait une approche plus pragmatique que celle utilisée actuellement et améliorerait la compréhension et, ultimement, les mesures utilisées par les particuliers et les systèmes.

\section{Conclusion}

Dans le cadre de la présente étude, des décideurs ont été consultés sur les méthodes de transfert de connaissances à adopter en ce qui concerne les données sur les blessures. Un certain nombre de questions soulevées par les participants sont pertinentes au transfert de connaissances efficace. Nous supposons que l'opinion des décideurs restera la même, peu importe le mandat qui leur est attribué, et que les résultats peuvent donc être appliqués à divers domaines. Il serait toutefois utile de corroborer les résultats à l'aide, par exemple, d'études réalisées auprès de décideurs concernés par d'autres maladies chroniques. Selon nous, ces résultats reflètent la pertinence de l'environnement politique et soulignent les diverses étapes du processus de prise de décisions stratégiques qui doivent être davantage étudié afin d'assurer la mise en application et l'utilisation optimales des connaissances découlant des recherches.

\section{Références}

1 Landry R, Amara N, Pablos-Mendes A, Shademani R, Gold I. The knowledge-value chain: a conceptual framework for knowledge translation in health. Bull World Health Organ. 2006;84:597-602.

2. Innvaer S, Vist G, Trommald M, Oxman A. Health policy-makers' perceptions of their use of evidence: a systematic review. J Health Serv Res Policy. 2002;7:239-44.

3. Dobbins M, Cockerill R, Barnsley J, Ciliska, D. Factors of the innovation, organization, environment, and individual that predict the influence five systematic reviews had on public health decisions. Int J Technol Asses. 2001;17:467-78.
4. Bowen S, Martens P, the Need to Know Team. Demystifying knowledge translation: learning from the community. J Health Serv Res Policy. 2005;10:203-11.

5. Lavis J, Ross S, Hurley J, et al. Examining the role of health services research in public policymaking. Milbank Q. 2002;80:125-54.

6. Lavis J, Robertson D, Woodside JM, et al. How can research organizations more effectively transfer research knowledge to decision makers? Milbank Q. 2003;81: 221-48.

7. Jacobson N, Butterill D, Goering P. Development of a framework for knowledge translation: understanding user context. J Health Serv Res Policy. 2003;8:94-99.

8. Elliott H, Popay J. How are policy makers using evidence? Models of research utilisation and local NHS policy making. J Epidemiol Commun Health. 2000;54:461-68.

9. Lomas J. Using linkage and exchange to move research into policy at a Canadian foundation. Health Affair. 2000;19:236-40.

10. Roos NP, Shapiro E. From research to policy: what have we learned? Med Care. 1999; 27:JS291-JS305.

11. Bryman A, Lewis-Beck MS, Liao TF, Parry KW, eds. Constant comparison. The Sage encyclopedia of social science research methods, Thousand Oaks, Californie, Sage, 2004:180-81.

12. Office of the Provincial Health Officer. Prevention of falls and injuries among the elderly, 2004. URL : http://www.health. gov.bc.ca/pho/pdf/falls.pdf.

13. MacNab YC, Kmetic A, Gustafson P, Shaps S. An innovative application of Bayesian disease mapping methods to patient safety research: the Canadian iatrogenic injury study. Stat Med. 2006;25:3960-980.

14. MacNab YC. Mapping disability-adjusted life years: a Bayesian hierarchical model framework for burden of disease and injury assessment. Stat Med. 2007;26:4746-769. 
15. Murray CJL, Lopez AD (dir.). The global burden of disease, Cambridge, Harvard University Press, 1996.

16. MacNab YC. Spline smoothing in Bayesian disease mapping. Environmetrics. 2007; 18:727-44.

17. Fraser I. Organizational research with impact: working backwards. Worldviews Evid Based Nurs. 2004;1:S52-S59.

18. Howlett M, Dunn C, (dir.). Policy development. In The handbook of public administration in Canada, Oxford University Press, 2003:173-91.

19. Howlett M, Ramesh M. Studying public policy: policy cycles and policy subsystems, Toronto, Oxford University Press, 1995.

20. Vingilis E, Hartford K, Schrecker T, Mitchell B, Lent B, Bishop J. Integrating knowledge generation with knowledge diffusion and utilization. Can J Public Health. 2003; 94:468-71.

21. Wong K, Gardner S, Bainbridge DB, Feightner K, Offord DR, Chambers LW. Tracking the use and impact of a community social report: where does the information go? Can J Public Health. 2000;91:41-45.

22. Kothari A, Birch S, Charles C. "Interaction" and research utilisation in health policies and programs: does it work? Health Policy. 2005;71:117-25

23. Hanney S, Gonzalez-Block M, Buxton M, Kogan M. The utilization of health research in policy-making: concepts, examples and methods of assessment. Health Res Policy Syst. 2003 Jan 13;1:2.

24. Reimer B, Sawka E, James D. Improving research transfer in the addictions field: a perspective from Canada. Subst Use Misuse. 2005;40:1707-720.

25. Belkhodja O, Amara N, Landry R, Ouimet, M. The extent and organizational determinants of research utilization in Canadian health services organizations. Sci Commun. 2007;28:377-417.

\section{Annexe}

\section{Protocole d'entrevue}

1. Veuillez décrire votre rôle en ce qui a trait à la prévention et au contrôle des blessures et à la planification, à la mise en œuvre et à l'élaboration des politiques concernant les blessures en Colombie-Britannique.

2. Veuillez décrire brièvement les données et les renseignements relatifs au fardeau des blessures en ColombieBritannique auxquels vous avez accès et que vous utilisez dans le cadre de vos fonctions.

3. Les données et les renseignements que vous utilisez actuellement sont-ils adéquats?

4. Quels sont les points forts et les points faibles des données et des renseignements actuellement disponibles?

5. (Montrez au participant les tableaux à l'aide d'un ordinateur portatif.) Veuillez examiner les tableaux présentés à l'écran. Ils contiennent des renseignements précis sur les variations des taux de mortalité, de morbidité liés aux blessures et sur le fardeau connexe. (Laisser le participant examiner les tableaux durant environ 10 à 15 minutes.) Quelles sont les données les plus importantes pour vous à la lumière de vos fonctions actuelles? Pour quelles raisons?

6. En vous référant encore une fois aux données du tableau, quelle serait la manière la plus efficace de présenter ces renseignements et de s'assurer que vous ou vos collègues les utilisez?

7. Si ces données vous étaient présentées comme vous l'avez décrit, de quelle manière les utiliseriez-vous?
8. Selon vous, ces données auraient-elles des répercussions directes sur l'établissement des priorités et les activités liées à l'allocation des ressources de votre (ministère ou organisation)? Veuillez préciser de quelle manière.

9. (Montrez au participant l'analyse géographique à l'aide de l'ordinateur portatif). Veuillez examiner la représentation géographique présentée à l'écran. Elle contient les mêmes renseignements qui étaient fournis dans le tableau présenté antérieurement. (Laisser le participant examiner la ou les carte(s) durant environ 10 à 15 minutes.) Cette manière de présenter les données est-elle efficace ou préférez-vous que les données soient présentées à l'aide d'un tableau? Veuillez préciser les raisons de votre choix.

10. En considérant que nous allons élaborer une stratégie de transfert de connaissances, qui permettra de déterminer la manière optimale de communiquer les renseignements aux décideurs, avez-vous d'autres suggestions qui pourraient aider à aller de l'avant?

Nous vous remercions sincèrement d'avoir participé à la présente entrevue. 